\title{
Effect of Micro- to Nanosize Inclusions upon the Thermal Conductivity of Powdered Composites with High and Low Interface Resistance
}

\author{
Muhammad Zain-ul-abdein,, Waqas S. Awan, ${ }^{1}$ Hassan Ijaz, ${ }^{1}$ Aqeel A. Taimoor, ${ }^{2}$ \\ Ayyaz Muhammad, ${ }^{2,3}$ and Sami ullah Rather ${ }^{2}$ \\ ${ }^{1}$ Mechanical Engineering Department, University of Jeddah, Jeddah, Saudi Arabia \\ ${ }^{2}$ Department of Chemical and Materials Engineering, King Abdulaziz University, Jeddah, Saudi Arabia \\ ${ }^{3}$ Institute of Chemical Engineering and Technology, University of Punjab, Lahore, Pakistan
}

Correspondence should be addressed to Muhammad Zain-ul-abdein; mzainulabdein@gmail.com

Received 17 June 2015; Accepted 28 September 2015

Academic Editor: Christian Brosseau

Copyright (C) 2015 Muhammad Zain-ul-abdein et al. This is an open access article distributed under the Creative Commons Attribution License, which permits unrestricted use, distribution, and reproduction in any medium, provided the original work is properly cited.

\begin{abstract}
Materials for thermal management application require better control over the thermophysical properties, which has largely been achieved by fabricating powdered composite. There are, however, several factors like filler volume fraction, shape morphology, inclusion size, and interfacial thermal resistance that limit the effective properties of the medium. This paper presents a methodology to estimate the effective thermal conductivity of powdered composites where the filler material is more conductive than the matrix. Only a few theoretical models, such as Hasselman and Johnson (HJ) model, include the effect of interfacial resistance in their formulation. Nevertheless, HJ model does not specify the nature of the interfacial thermal resistance. Although Sevostianov and Kachanov (SK) method takes care of interface thickness, they, on the other hand, have not taken into account the interfacial resistance due to atomic imperfections. In the present work, HJ model has been modified using SK method and the results were compared with experimental ones from the literature. It has been found that the effect of interfacial resistance is significant in highly resistive medium at microscale compared to nanoscale, such as $\mathrm{Cu}$ /diamond system, while, in a highly conductive medium, like bakelite/graphite system, the effect of shape factor is more significant than interfacial thermal resistance.
\end{abstract}

\section{Introduction}

Thermal conductivity is one of the fundamental properties of key importance in the energy applications where variable (fast/slow) heat dissipation/storage is often required. Although metals and alloys provide a large range of conductivities to be used in several electronic and mechanical appliances, the quest for the search of new composite materials with modifiable properties has still been unquenched. In addition to fabricating the two-phase composites with different inclusions, like metal matrix composite (MMC), polymer matrix composite (PMC), and so forth, the researchers have shown growing inclination towards manipulating the inclusion size. It is, therefore, of interest to be able to observe the effective conductive properties of a given composite from micro- to nanoregimes.

In the current framework, Clausius (1879) [1] and Maxwell (1884) [2] introduced one of the earliest models based upon self-consistent scheme (SCS). Both models attempted to predict the effective properties of a two-phase medium where the inclusions, in dilute concentration, were treated as isolated particles immersed into a given matrix. Bruggeman (1935) [3], later on, implemented an incremental scheme called differential scheme (DS) or effective medium scheme (EMS). The basic idea was to introduce the second phase particle into a homogenous matrix in small increments and estimate the overall properties of the resulting medium. Nevertheless, the very first upper and lower bounds based 
only upon volume fractions of matrix and inclusion were formulated by Wiener (1912) [4]. The Wiener upper bound (UB) is still widely used by the researchers as the "rule of mixtures." Assuming macroscopically isotropic behavior of the composite, Hashin and Shtrikman (1962) [5] defined a more conservative set of upper and lower bounds. For a composite with spherical inclusions, the Hashin-Shtrikman (HS) lower bound (LB) coincides with Maxwell and Mori and Tanka (1973) [6] models. Mori-Tanaka (MT), however, also introduced depolarization factors in their formulation to accommodate the prolate and oblate shape morphologies. Similar to the Kanaun and Levin (1994) [7] scheme, MT scheme is merely a variant of effective field (EF) method which treats the inclusion as an isolated particle and assumes the presence of an effective field over each inhomogeneity (see Markov (2000) [8] for a detailed review). Hamilton and Crosser (1962) [9] modified Maxwell model to incorporate the shape factor $(n=3 / \psi)$ for nonspherical particles, where the sphericity $(\psi)$ is the ratio of the surface area of sphere with the same volume of the particle to the surface area of the particle. Using $\psi=1$ for a spherical inclusion regenerates the Maxwell model. Likewise, Zimmerman (1996) [10] exploited Maxwell and Bruggeman methods and introduced an aspect ratio and conductivity ratio dependent parameter $(\beta)$ for elliptical inhomogeneities.

Most of the above mentioned models assume ideal contact between the inclusion and the matrix, which implies that the heat flow across the interface remains continuous. In reality, however, the interface, regardless of its nature, is nonideal and leads to a discontinuous temperature field across the boundary due to interfacial thermal resistance, also known as Kapitza's resistance (after Kapitza (1941) [11]). In order to deal with interface behavior, Kanaun (1984) [12] introduced the concept of singular inclusion in the elasticity context. From the perspective of thermal conductivity, the nonideal contact may be assumed as either "superconductive" or "resistive." A superconducting or resisting singular inclusion requires that its conductivity $\left(k_{s}\right)$ be $k_{s} \rightarrow \infty$ or $1 / k_{s} \rightarrow \infty$, respectively, provided that one of the inclusion's dimensions is of the order $\delta \rightarrow 0$. Torquato and Rintoul (1995) [13] also defined conductive and resistive contacts in relation to the conductive properties of the interphase layer between the matrix and inclusion. Detailed discussions on conductive/resistive interfaces may also be found in the works of Benveniste and Miloh (1986) [14], Benveniste (1987) [15], and Chiew and Glandt (1987) [16].

In the present work, a method based on Hasselman and Johnson (1987) [17] and Sevostianov and Kachanov (2007) [18] models has been proposed to predict the thermal conductivity of particulate filled composites with high and low interfacial thermal resistance as functions of filler volume fractions, inclusion size, shape factor, and conductivity of interphase layer. The effect of particle size ranging from micro- to nanoscale has been investigated in the highly conductive and resistive media. It will be shown with the help of case studies that the inclusion size behaves differently in response to the interfacial resistance.

\section{Theoretical Model}

2.1. Effects of Shape Factor and Interfacial Resistance. In their work, Hasselman and Johnson [17] addressed the nonideal contacts in terms of interfacial thermal resistance $(R)$ and derived a mathematical model for spherical morphology of particles. The model states

$$
\begin{aligned}
k_{\mathrm{eff}} & =k_{m} \\
& \frac{2\left(k_{f} / k_{m}-1-\left(k_{f} / r_{o}\right) R\right) \phi_{f}+k_{f} / k_{m}+2+2\left(k_{f} / r_{o}\right) R}{\left(1-k_{f} / k_{m}+\left(k_{f} / r_{o}\right) R\right) \phi_{f}+k_{f} / k_{m}+2+2\left(k_{f} / r_{o}\right) R},
\end{aligned}
$$

where $k_{m}$ and $k_{f}$ are thermal conductivities of matrix and filler (inclusion) in $\mathrm{W} \cdot \mathrm{m}^{-1} \cdot \mathrm{K}^{-1}$, respectively, $r_{o}$ is the filler radius in $\mathrm{m}, \phi_{f}$ is the filler volume fraction, $R$ is the interfacial resistance in $\mathrm{W}^{-1} \cdot \mathrm{m}^{2} \cdot \mathrm{K}$, and $k_{\text {eff }}$ is the effective thermal conductivity of the composite. $R$, by definition, is an inverse of thermal conductance $(C)$; that is, $R=1 / C$. Note that $\mathrm{HJ}$ model is valid for any system of units, the SI units are mentioned here only for the reader's convenience. Jiajun and Xiao-Su (2004) [19] modified the HJ model (1) in order to accommodate the nonspherical morphology of the particles. They actually used Hamilton and Crosser [9] shape factor parameter $(n=3 / \psi)$, where $\psi$ is the sphericity, as described earlier. The modified HJ, therefore, takes the following form:

$$
k_{\mathrm{eff}}=k_{m} \frac{k_{f}\left(1+(n-1)\left(k_{m} / r_{o}\right) R\right)+(n-1) k_{m}+(n-1) \phi_{f}\left(k_{f}\left(1-\left(k_{m} / r_{o}\right) R\right)-k_{m}\right)}{k_{f}\left(1+(n-1)\left(k_{m} / r_{o}\right) R\right)+(n-1) k_{m}-\phi_{f}\left(k_{f}\left(1-\left(k_{m} / r_{o}\right) R\right)-k_{m}\right)} .
$$

Note that (2) reduces to (1) for spherical inclusions, that is, $\psi=1$, and also that the $\mathrm{HJ}$ model reproduces the Maxwell model for ideal contact when $R=0$ (or $C=\infty$ ). Conversely, for nonideal contacts, the interfacial resistance must be nonzero and the conductance finite. It should also be mentioned that increasing filler fractions yield either decreasing $k_{\text {eff }}$ when $k_{m}>C \cdot r_{o}$ or increasing $k_{\text {eff }}$ when $k_{m}<C \cdot r_{o}$. Consequently, the interfacial thermal resistance would be substantially large in the former case, as in resistive composites, and vanishingly small in the latter case, similar to the superconductive composites.

2.2. Effect of Inclusion Size: Micro- to Nanoscale. Sevostianov and Kachanov [18] recently modified an incremental scheme formulated by Shen and $\mathrm{Li}$ (2005) [20] so as to incorporate the effect of interface layer thickness $(h \sim 1-2 \mathrm{~nm}$ [21]) upon the overall properties of the material at nanoscale. It is understood that in the microregime $h \ll r_{o}$; however, in 


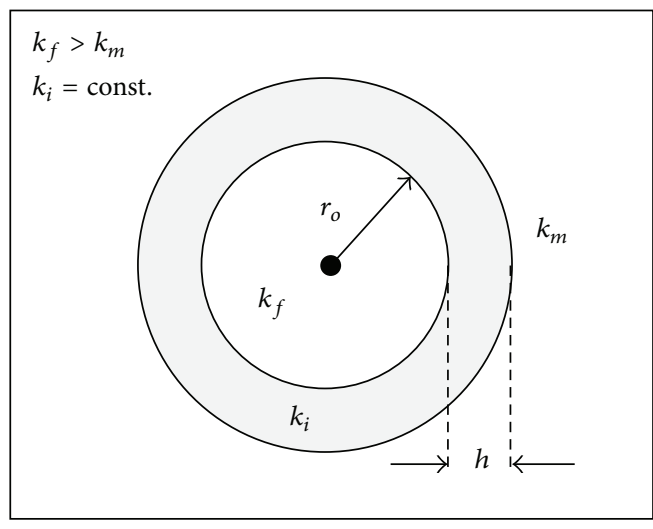

(a)

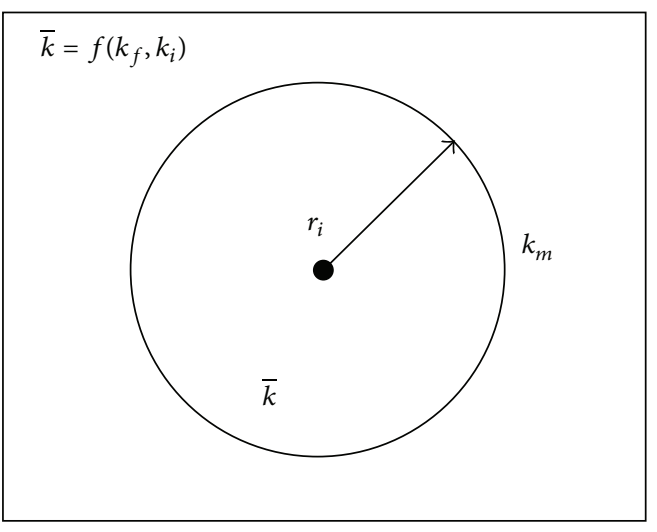

(b)

FIGURE 1: SK model: (a) interface zone; (b) equivalent homogeneous inclusion.

case of a nanoinclusion the outer radius $r_{o}$ is comparable to the interface thickness; that is, $h \approx r_{o}$. The basic idea was to incrementally add the interface thickness to the spherical inclusion and homogenize at each step until $r_{o}+h=$ $r_{i}$, where $r_{i}$ is the outer radius of the interface layer (see Figure 1). Given that the current radius $(r)$ is $r_{o}<r<r_{i}$, the conductivity across the interface $k_{i}=k_{i}(r)$ and the conductivity of the equivalent homogeneous radius $r_{i}$ is $\bar{k}=$ $\bar{k}\left(r_{i}\right)$, a differential equation, with initial condition $\bar{k}\left(r_{o}\right)=$ $k_{f}$, of the following form may be written for the equivalent homogeneous inclusion:

$$
\frac{d \bar{k}}{d r}=-\frac{1}{r} \frac{\left(\bar{k}(r)-k_{i}(r)\right)\left(\bar{k}(r)+2 k_{i}(r)\right)}{k_{i}(r)}
$$

Setting $r_{o}+h=r_{i}=r$ for the simplest case of a homogeneous interface zone, $k_{i}(r)=k_{i}=$ const, integration of (3) yields

$$
\begin{aligned}
& \bar{k}\left(r_{i}\right) \\
& =k_{i}\left[\frac{2+\left(1+h / r_{o}\right)^{3} \cdot\left(\left(2\left(k_{i} / k_{f}\right)+1\right) /\left(1-\left(k_{i} / k_{f}\right)\right)\right)}{\left(1+h / r_{o}\right)^{3} \cdot\left(\left(2\left(k_{i} / k_{f}\right)+1\right) /\left(1-\left(k_{i} / k_{f}\right)\right)\right)-1}\right] .
\end{aligned}
$$

For a general solution of (3) in terms of hypergeometric functions the reader is referred to Sevostianov and Kachanov [18]. The estimation of effective thermal conductivity of the composite requires placement of the equivalent homogeneous inclusion in the effective field (EF) of heat flux (Levin [22]; Kanaun [23]; and Kanaun and Levin [7]). Based upon above discussion, Sevostianov-Kachanov (SK) modified the
HS bounds and EF method to calculate the $k_{\text {eff }}$ as in the following:

$$
\begin{aligned}
& k_{m}\left[1+\frac{3 \phi^{\prime}\left(\bar{k}-k_{m}\right)}{3 k_{m}+\left(1-\phi^{\prime}\right)\left(\bar{k}-k_{m}\right)}\right] \leq k_{\mathrm{eff}} \\
& \leq \bar{k}\left[1-\frac{3\left(1-\phi^{\prime}\right)\left(\bar{k}-k_{m}\right)}{3 \bar{k}-\phi^{\prime}\left(\bar{k}-k_{m}\right)}\right] \quad \text { (HS bounds) } \\
& k_{\mathrm{eff}}=k_{m}\left[1+\frac{3 \phi^{\prime}\left(\bar{k}-k_{m}\right)}{3 k_{m}+\left(1-\phi^{\prime}\right)\left(\bar{k}-k_{m}\right)}\right]
\end{aligned}
$$

(Kanaun-Levin EF method),

where $\phi^{\prime}$ is the filler volume fraction including interface layer. Note that the Kanaun-Levin EF method coincides with the Maxwell model and HS lower bound.

2.3. Proposed Scheme: Modified HJ and SK Method. It has been assumed throughout that $k_{f}>k_{m}$, which in case of ideal contact would lead to $k_{f}>k_{\text {eff }}>k_{m}$. Nonideal contact, however, requires that $k_{i}$ be a function of $k_{m}$ in the limit $r_{o}<$ $r<r_{i}$. Subject to the condition of a homogeneous interface zone $\left(k_{i}=\right.$ const), an interface with very high resistance may acquire conductivity $k_{i}=0.01 k_{m}$. Likewise, for very low interfacial resistance, $k_{i}=0.99 k_{m}$. It has been pointed out earlier that the interface thickness $(h)$ is of the order of 1-2 $\mathrm{nm}$, which, at atomic level, means placing almost 10 20 average sized atoms side by side in the thickness direction. Here, the interface would be comprised of not only filler and matrix atoms, but also the interatomic defects like vacancies, and so forth. It further implies that a higher concentration of defects would result in an increased interfacial resistance and vice versa (Figure 2). This effect may be incorporated in terms of $R$ as in modified HJ model (2). The SK method may now be employed to calculate $k_{\text {eff }}$ from (2) as follows:

$$
k_{\mathrm{eff}}=k_{m} \frac{\bar{k}\left(1+(n-1)\left(k_{m} / r_{i}\right) R\right)+(n-1) k_{m}+(n-1) \phi^{\prime}\left(\bar{k}\left(1-\left(k_{m} / r_{i}\right) R\right)-k_{m}\right)}{\bar{k}\left(1+(n-1)\left(k_{m} / r_{i}\right) R\right)+(n-1) k_{m}-\phi^{\prime}\left(\bar{k}\left(1-\left(k_{m} / r_{i}\right) R\right)-k_{m}\right)}
$$




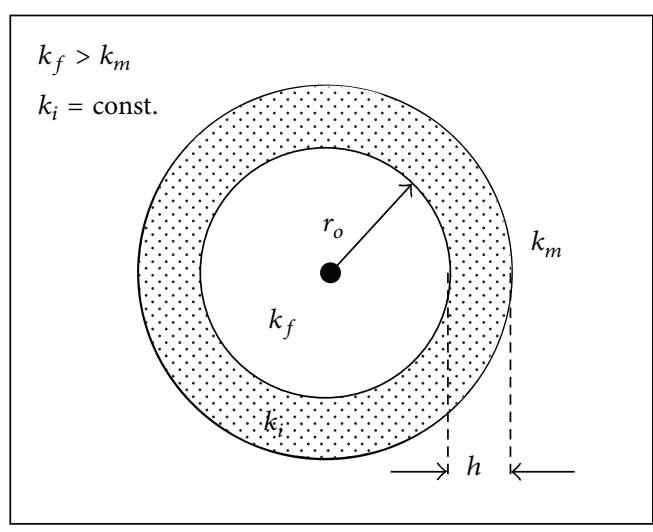

(a)

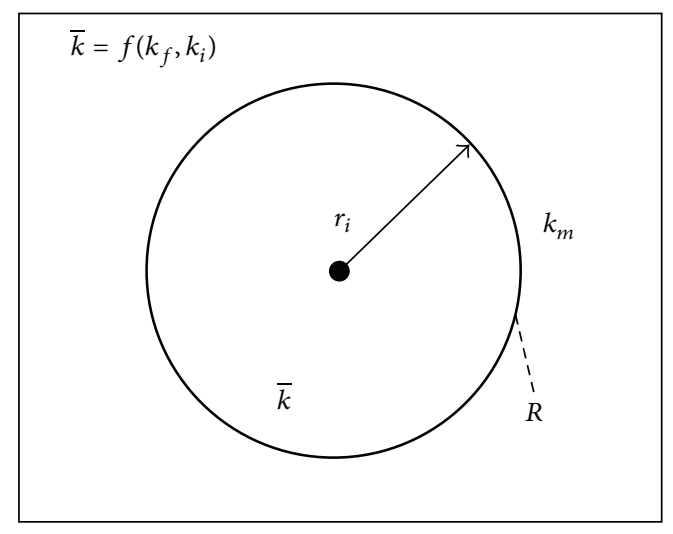

(b)

FIGURE 2: Current model: (a) interface zone + interatomic defects; (b) equivalent homogeneous inclusion + interfacial resistance.

where $\bar{k}$ is a function of $k_{i}, k_{f}, r_{o}$, and $h$ defined previously in (4). Note that the above equation estimates effective conductivity considering volume fraction of filler with interface layer $\left(\phi^{\prime}\right)$, shape factor $(n)$, filler, matrix, and interface conductivities $\left(k_{f}, k_{m}, k_{i}\right)$, particle size including interface thickness $\left(r_{i}\right)$, and interfacial resistance $(R)$. Also note that the factor $h / r_{o}$ is negligibly small at microlevel while significantly large at nanoscale. It must be mentioned that a size of $0.1 \mu \mathrm{m}$ was assumed to be the lower limit of micro- and upper limit of nanoinclusions; that is, microscale $>0.1 \mu \mathrm{m}>$ nanoscale.

\section{Case Studies}

The application of the above method will now be presented in the context of two case studies: (i) a metal matrix $\mathrm{Cu}$ /diamond composite with decreasing conductivity as a function of diamond volume fraction (Hanada et al. (2004) [24]) and (ii) a polymer matrix graphite/bakelite composite with increasing conductivity as a function of graphite volume fraction (Azeem and Zain-Ul-abdein (2012) [25]). The two composites were chosen as representatives of highly resistive and conductive systems.

3.1. Copper/Diamond Composite: High Interfacial Resistance. In the literature, several copper/diamond composites have been reported to yield decreasing thermal conductivity with increasing diamond volume fraction due to high interfacial resistance (Hanada et al. [24], Kang et al. [26], Shen et al. [27], and Raza and Khalid [28]). Given that the thermal conductivities of $\mathrm{Cu}$ and diamond are $k_{m}=400 \mathrm{Wm}^{-1} \mathrm{~K}^{-1}$ and $k_{f}=2000 \mathrm{Wm}^{-1} \mathrm{~K}^{-1}$, respectively, (4) can be used to draw $\bar{k} / k_{f}$ as a function of $h / r_{o}$ for different values of $k_{i}$ while assuming $h=2 \mathrm{~nm}$ (see Figure 3 ). The curve shows that as $r_{o}$ varies from nano- to microsize; $\bar{k}$ approaches $k_{f}$. Moreover, increase in $k_{i}$ from $0.01 k_{m}$ to $0.99 k_{m}$ results in a significant improvement of $\bar{k}$ values. Since the interface is highly resistive, $k_{i}=0.01 k_{m}$ is more likely to yield realistic

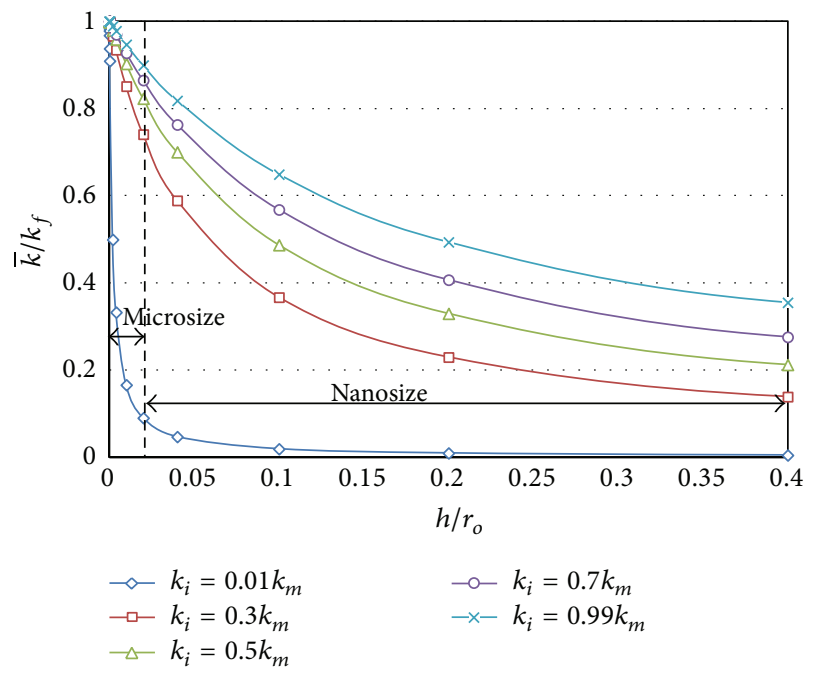

FIGURE 3: Cu/diamond composite system: $\bar{k} / k_{f}$ as a function of $h / r_{o}$ (4).

conductivity values for the $\mathrm{Cu} /$ diamond system within the nano- and microregions.

Applying the above condition to the HS lower bound in (5) would result in the effective thermal conductivity of composite. Figure 4 illustrates the calculated thermal conductivity as a function of filler (diamond) volume fraction as well as the micro- and nanoregimes. Note that the experimental conductivities for $\mathrm{Cu} /$ diamond system as measured by Hanada et al. [24], who actually used irregular shaped diamond particles with an average size of $7.7 \mu \mathrm{m}$, are also presented. Figure 4, however, shows that the measured conductivities lie within the nanoregime and, contrary to the experimental findings, a filler size of $7.7 \mu \mathrm{m}$ should yield increasing thermal conductivity (see the dotted line labeled $7.7 \mu \mathrm{m}$ ). Reason for this discrepancy is that the HS lower bound assumes spherical inclusions and does not take into account the interfacial resistance $(R)$. It, therefore, requires 


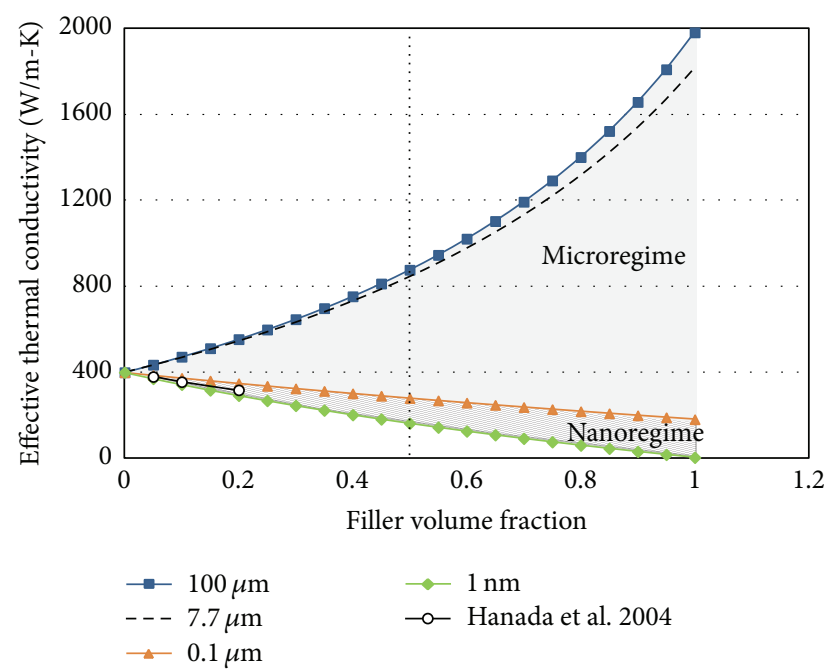

Figure 4: Cu/diamond composite system: $k_{\text {eff }}$ using HS Lower Bound $\left((5), k_{i}=0.01 k_{m}\right)$.

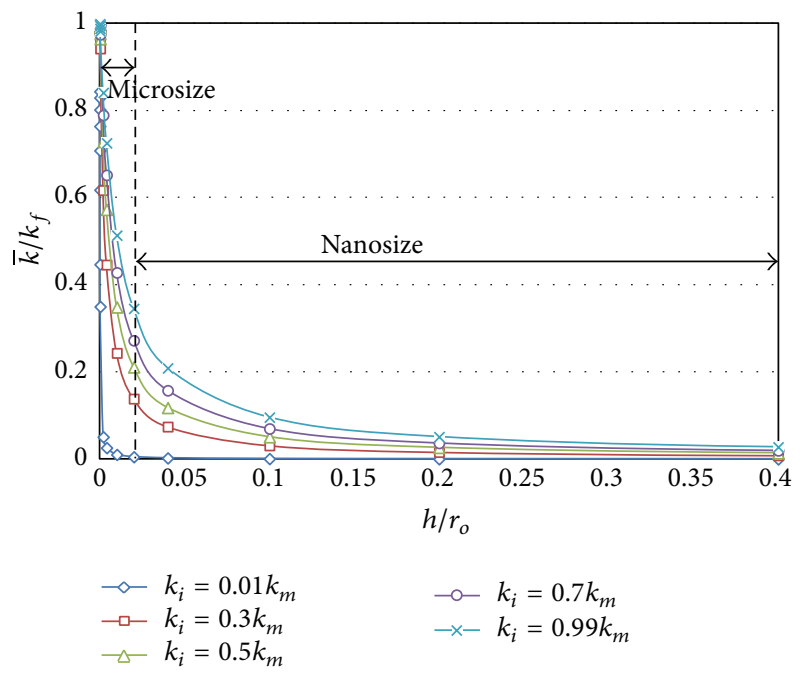

FIGURE 5: Bakelite/graphite composite system: $\bar{k} / k_{f}$ as a function of $h / r_{o}(4)$.

modification to incorporate the effect of $R$, which will be treated in Section 4.

3.2. Bakelite/Graphite Composite: Low Interfacial Resistance. In a recent study, Azeem and Zain-Ul-Abdein [25] have investigated effective thermal conductivity of a polymer matrix bakelite/graphite composite. Graphite, added in increasing volume fractions, was found to yield improved properties of the system. A similar observation was also made by Ling et al. [29], where they used expanded graphite to enhance the thermal conductivity of an organic phase change material. Figure 5 correlates $\bar{k} / k_{f}$ with $h / r_{o}$ against different $k_{i}$ values using (4) and assuming $h=2 \mathrm{~nm}, k_{m}=1.4 \mathrm{Wm}^{-1} \mathrm{~K}^{-1}$ for bakelite, and $k_{f}=130 \mathrm{Wm}^{-1} \mathrm{~K}^{-1}$ for graphite. Note that an overall trend of the curves is identical to that of Figure 3;

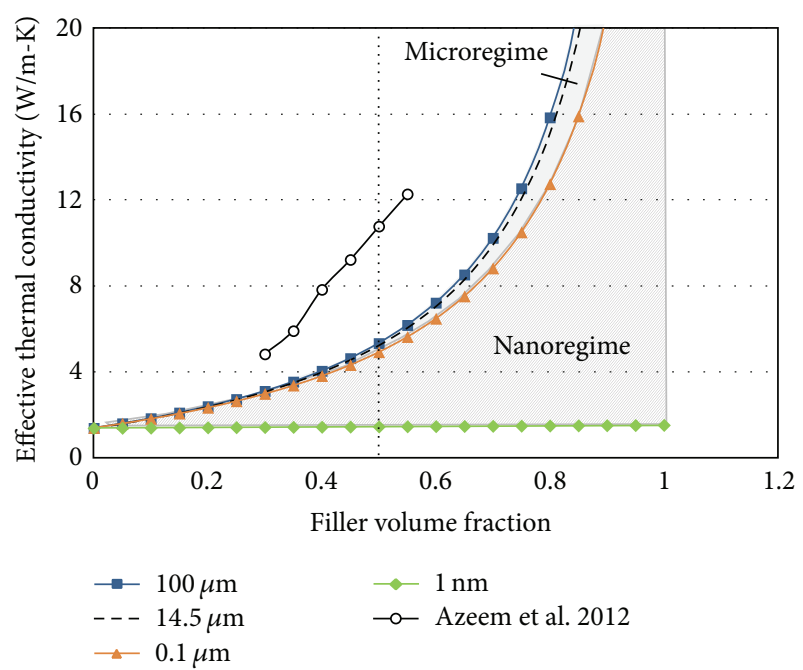

FIGURE 6: Bakelite/graphite composite system: $k_{\text {eff }}$ using HS lower bound $\left((5), k_{i}=0.99 k_{m}\right)$.

that is, the $\bar{k}$ increases with increasing $r_{o}$ and also when $k_{i}$ varies from $0.01 k_{m}$ to $0.99 k_{m}$. However, it may also be noticed that the slopes of the curves in Figure 5 are steeper, at least within the limits of microscale, than the corresponding curves in Figure 3. This is simply a result of relatively higher $k_{f} / k_{m}$ ratio in bakelite/graphite composite (Figure 5) than in $\mathrm{Cu}$ /diamond composite (Figure 3). Since the interface was assumed to have very low resistance (or high conductance), it may be expected that $k_{i}=0.99 k_{m}$ would predict the acceptable results.

HS lower bound may now be obtained using (5) and conductivities of bakelite and graphite. Figure 6 summarizes the effective thermal conductivities of the composite within the micro- and nanoregimes as a function of filler (graphite) volume fraction. The experimental values of the bakelite/graphite system from Azeem and Zain-Ul-Abdein [25] are also shown for comparison. The authors used graphite powder of irregular shape morphology with an average size of $14.5 \mu \mathrm{m}$. In comparison to experimental results, it may be observed that HS lower bound underestimates the conductivity values. Clearly, the inherent assumptions that the inclusions are spherical and there is no interfacial resistance $(R)$ are insufficient to reproduce the experimental findings (see the dotted line labeled $14.5 \mu \mathrm{m}$ in Figure 6). The modification to incorporate the effects of interfacial resistance and inclusion shape morphology is discussed in the subsequent section.

\section{Results and Discussion}

4.1. Effect of Particle Size: HS Lower Bound. Although the HS lower bound could not reproduce the experimental results at a given particle size, it may, however, be observed that the nanoregime for the resistive (Figure 4) as well as conductive (Figure 6) media is overshadowed by the microregime. It will be shown, shortly, that this behavior does not change 
even with the introduction of shape factor $(n)$ and interfacial resistance $(R)$.

It should also be noticed that in a resistive medium the conductivity values span over a large range for the microsized inclusions, while nanosize limits the thermal properties within a very short range. For instance, consider the $\mathrm{Cu} /$ diamond composite with a volume fraction of 0.5 for each. Here, the difference in conductivities was found to be $\Delta k_{\text {micro }}=$ $k_{100 \mu \mathrm{m}}-k_{0.1 \mu \mathrm{m}} \approx 597 \mathrm{Wm}^{-1} \mathrm{~K}^{-1}$ and $\Delta k_{\text {nano }}=k_{0.1 \mu \mathrm{m}}-$ $k_{1 \mathrm{~nm}} \approx 117 \mathrm{Wm}^{-1} \mathrm{~K}^{-1}$ within the micro- and nanoregimes, respectively. This shows that the former differs from the latter by a factor of almost 5 . This behavior is a result of very low conductivity of the interface, $k_{i}=0.01 k_{m}$, which leads to a rapid decrease in effective properties of the medium as $r_{o}$ varies from micro- to nanoscale.

On the contrary, in a conductive medium the nanosize inclusions offer large range of effective properties, whereas microsize inclusions confine the conductivity values to a very small range. For example, for the bakelite/graphite composite with a volume fraction of 0.5 for each constituent, the conductivity difference within micro- and nanoregimes was $\Delta k_{\text {micro }}=k_{100 \mu \mathrm{m}}-k_{0.1 \mu \mathrm{m}} \approx 0.42 \mathrm{Wm}^{-1} \mathrm{~K}^{-1}$ and $\Delta k_{\text {nano }}=k_{0.1 \mu \mathrm{m}}-k_{1 \mathrm{~nm}} \approx 3.45 \mathrm{Wm}^{-1} \mathrm{~K}^{-1}$, respectively. Here, the latter is 8 times more than the former. Obviously, a relatively conductive interface zone, $k_{i}=0.99 k_{m}$, would not allow significant decrease in properties at least at microscale. Within nanoregime, however, the more the $r_{o}$ decreases, the more the interface thickness $(h)$ becomes effective, which leads to a large span of properties.

4.2. Effect of Interfacial Thermal Resistance: Modified HJ Model. It has been pointed out earlier that the shape factor, $n=3 / \psi$, where $\psi=1$ for spherical and $\psi=0.9$ for cuboctahedral particles. The case studies under consideration, however, use random particles of graphite and diamond. In their work, Azeem and Zain-Ul-Abdein [25] determined that $\psi=0.4$ for irregular shape of graphite inclusions. Since Hanada et al. [24] did not mention the shape factor for their irregular diamond particles, the sphericity was assumed identical to that of graphite particles; that is, $\psi=0.4$.

4.2.1. Case I: High Interfacial Resistance. Considering $k_{i}=$ $0.01 k_{m}$ and $\psi=0.4$ for highly resistive $\mathrm{Cu}$ /diamond composite, (7) may be used to calculate the effective conductivity of the composite as a function of filler volume fraction over the entire range of micro- to nanosized particles (see Figure 7). Note that (7) also requires definition of $R$, such that the experimental values could be approximated. A value of $R$ $=3 \times 10^{-7} \mathrm{~m}^{2} \cdot \mathrm{K} \cdot \mathrm{W}^{-1}$ was found to reproduce experimental values to a reasonable agreement. It may be observed that (7) converges to HS lower bound for $R=0$ and $\psi=1$, that is, when the interfacial resistance is ignored and the inclusions are spherical. In the typical $\mathrm{Cu} /$ diamond composites, the $R$ values range from $1 \sim 3 \times 10^{-8} \mathrm{~m}^{2} \cdot \mathrm{K} \cdot \mathrm{W}^{-1}$ (Kang et al. [26], Shen et al. [27]) depending upon the diamond shape morphology and interface characteristics. The $R$-value identified in this work is almost 10 times more than the one found in the literature. This simply means that the $\mathrm{Cu} /$ diamond system

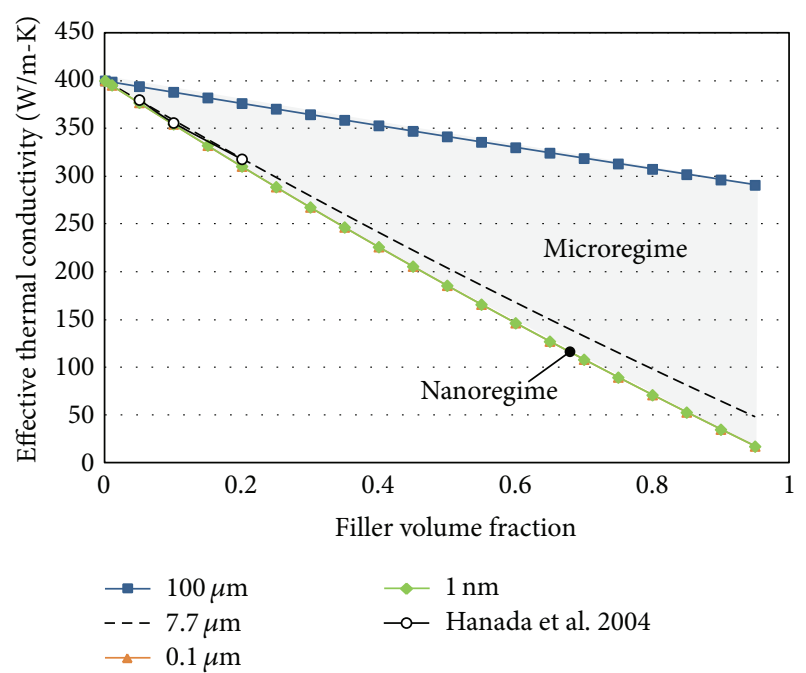

Figure 7: $\mathrm{Cu}$ /diamond composite system: $k_{\text {eff }}$ using modified $\mathrm{HJ}$ model $\left((7), R=3 \times 10^{-7} \mathrm{~m}^{2} \cdot \mathrm{K} \cdot \mathrm{W}^{-1}, k_{i}=0.01 k_{m}\right)$.

under consideration exhibits extremely high interfacial resistance. Or, in other words, as $R \rightarrow 0$ the interface becomes more conductive and less resistive. Probable reasons for high resistance are the imperfections at the interface including air voids and decohesion (see Raza and Khalid [28] for details).

A comparison of Figures 4 and 7 indicates that increase in $R$ leads to significant decrease in conductivity both within micro- and nanoregimes. It may be observed from Figure 7 that the experimental conductivities are at the limit of nanoscale. In addition, at such a high value of $R(3 \times$ $10^{-7} \mathrm{~m}^{2} \cdot \mathrm{K} \cdot \mathrm{W}^{-1}$ ) and a constant $\psi=0.4$, the effective conductivity of the composites is almost independent of the inclusion size at least within the nanoregime. Briefly, a resistive interface dominates the effect of both the particle size and shape.

4.2.2. Case II: Low Interfacial Resistance. Now considering $k_{i}=0.99 k_{m}$ and $\psi=0.4$ for highly conductive bakelite/graphite composite, (7) may be exploited to estimate the effective thermal conductivity of the medium as a function of filler volume fraction (see Figure 8). Here the value of $R$ has been identified as $1 \times 10^{-10} \mathrm{~m}^{2} \cdot \mathrm{K} \cdot \mathrm{W}^{-1}$ so as to yield the experimental values reported by Azeem and Zain-UlAbdein [25]. The observation that $R$ is quite close to 0 and is almost $3 \times 10^{-4}$ times smaller than that of $\mathrm{Cu} /$ diamond composite indicates the presence of almost perfect interface with extremely low resistance. In fact, any further diminution of $R$-value does not bring about any significant change in the conductivity values. This also means that, unlike the metal matrix $\mathrm{Cu}$ /diamond composite, there is a very less probability of interfacial defects such as air gaps and decohesion in case of polymer matrix bakelite/graphite composite.

A comparative analysis of Figures 6 and 8 suggests that although there is not much difference between the evolution of conductivity values within the micro- and nanoregimes using HS lower bound or modified HJ model, the latter helps in identifying the role of inclusion shape factor and interfacial 


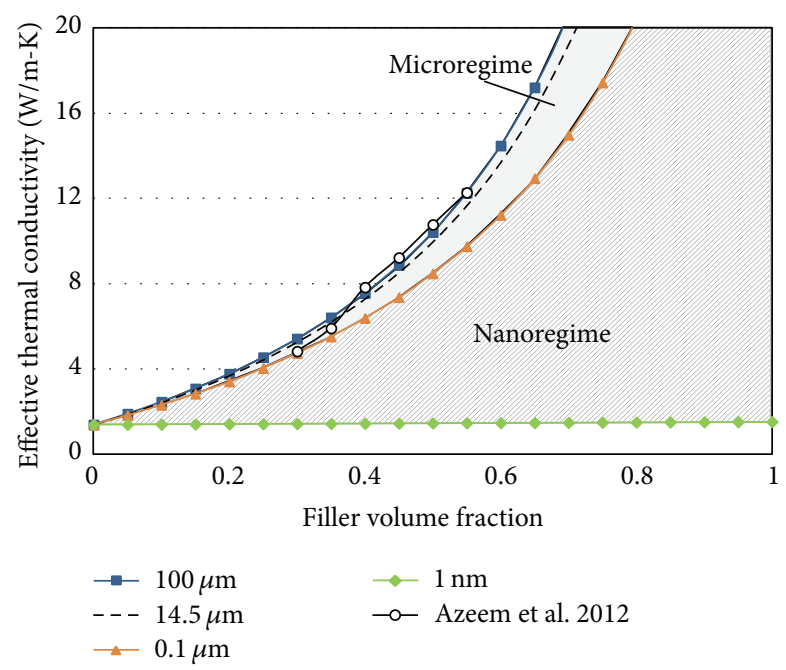

FIGURE 8: Bakelite/graphite composite system: $k_{\text {eff }}$ using modified $\mathrm{HJ}$ model $\left((7), R=1 \times 10^{-10} \mathrm{~m}^{2} \cdot \mathrm{K} \cdot \mathrm{W}^{-1}, k_{i}=0.99 k_{m}\right)$.

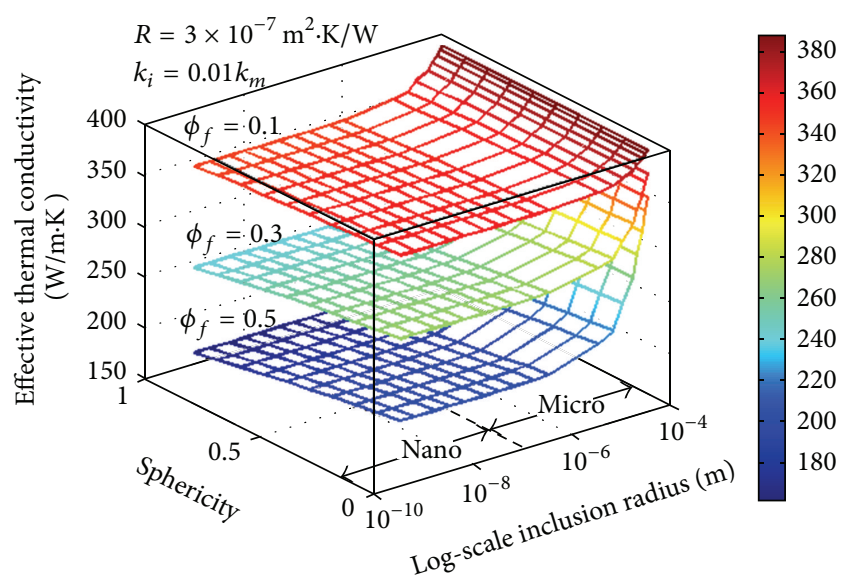

Figure 9: $\mathrm{Cu} /$ diamond composite system: $k_{\mathrm{eff}}$ as a function of $\phi_{f}, \psi$, and $r_{o}$.

thermal resistance more precisely. To summarize, the effect of shape factor is more pronounced in composites with low interfacial resistance.

4.3. Effect of Shape Factor. Since shape factor $(n)$ is a function of sphericity $(\psi)$ such that $0<\psi \leq 1$, it is relatively convenient to represent effective thermal conductivity as a function of sphericity. It is also of interest to observe simultaneously the effect of inclusion radius $\left(r_{o}\right)$ at micro- and nanoscales. Figures 9 and 10 illustrate the effects of both the sphericity and filler radius upon the thermal conductivity of $\mathrm{Cu}$ /diamond and bakelite/graphite composites, respectively, with filler volume fractions of $\phi_{f}=0.1,0.3$, and 0.5 .

In case of thermally resistive medium $\left(k_{i}=0.01 k_{m}\right.$, $R=3 \times 10^{-7} \mathrm{~m}^{2} \cdot \mathrm{K} \cdot \mathrm{W}^{-1}$ ), it may be noticed that the thermal conductivity is less responsive to the change in shape factor at a given volume fraction within the microregime than the nanoregime (Figure 9). Consider, for example, the extreme

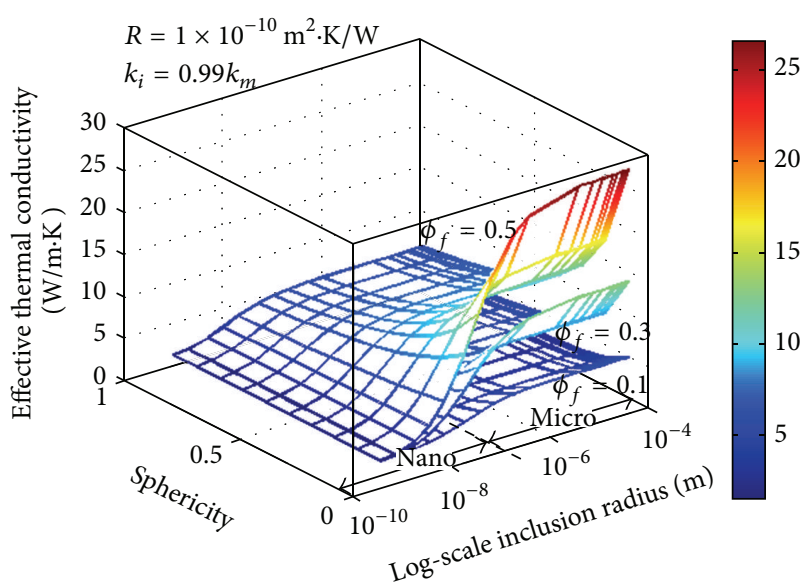

FIGURE 10: Bakelite/graphite composite system: $k_{\text {eff }}$ as a function of $\phi_{f}, \psi$, and $r_{o}$.

case when $\phi_{f}=0.5$, for $r_{o}=100 \mu \mathrm{m}$, the $k_{\text {eff }}$ varies from $339.8 \mathrm{~W} \cdot \mathrm{m}^{-1} \cdot \mathrm{K}^{-1}($ at $\psi=1)$ to $342.4 \mathrm{~W} \cdot \mathrm{m}^{-1} \cdot \mathrm{K}^{-1}($ at $\psi=0.1)$, whereas for $r_{o}=1 \mathrm{~nm}$ the $k_{\text {eff }}$ changes from $160 \mathrm{~W} \cdot \mathrm{m}^{-1} \cdot \mathrm{K}^{-1}$ (at $\psi=1$ ) to $196.6 \mathrm{~W} \cdot \mathrm{m}^{-1} \cdot \mathrm{K}^{-1}$ (at $\psi=0.1$ ).

Nevertheless, the thermally conductive medium $\left(k_{i}=\right.$ $0.99 k_{m}, R=1 \times 10^{-10} \mathrm{~m}^{2} \cdot \mathrm{K} \cdot \mathrm{W}^{-1}$ ) showed strong dependency of effective properties upon the shape factor as well as filler volume fraction (Figure 10). For instance, it may be noticed that when $\phi_{f}=0.5$ and $r_{o}=100 \mu \mathrm{m}$ the change in $k_{\text {eff }}$ ranges from 5.3 to $26.7 \mathrm{~W} \cdot \mathrm{m}^{-1} \cdot \mathrm{K}^{-1}$ for the corresponding values of $\psi$ from 1 to 0.1 , while for $r_{o}=1 \mathrm{~nm}$ the $k_{\text {eff }}$ does not change at least up to two significant digits; that is, $k_{\text {eff }}=1.4 \mathrm{~W} \cdot \mathrm{m}^{-1} \cdot \mathrm{K}^{-1}$ (at $\psi=0.1 \sim 1$ ).

\section{Conclusions}

Based upon the above investigation it may be concluded that the composite material for thermal management applications can offer wide range of properties that depend upon factors like volume fractions of the matrix and filler, shape morphology, and particle size. In addition, the properties of interface zone play a decisive role in limiting the behavior of composite within the limits of both the micro- and nanoregimes. The proposed scheme, in comparison to the published results, successfully estimates the effective properties for the extreme cases of binary composites with high and low interfacial resistance. Compared to nanoparticles, the contribution of microsize inclusions is less affected by the interface zone due to very small interface thickness to inclusion radius ratio. However, unlike the latter, the effect of the former is less influenced by the shape morphology or sphericity of the inclusions.

\section{Conflict of Interests}

The authors declare that there is no conflict of interests regarding the publication of this paper. 


\section{References}

[1] R. Clausius, Die Mechanische Behandlung der Electricität, Vieweg, Braunshweig, Germany, 1879.

[2] J. C. Maxwell, A Treatise on Electricity and Magnetism, Dover Publications, New York, NY, USA, 3rd edition, 1884.

[3] D. A. G. Bruggeman, "Calculation of various physical constants in heterogeneous substances. I. Dielectric constants and conductivity of composites from isotropic substances," Annalen der Physik, vol. 24, no. 5, pp. 636-679, 1935 (German).

[4] O. Wiener, "Die theorie des Mischkorpers fur das feld des stationaaren stromung. Erste abhandlung die Mttelswertsatze fur kraft," Abhandlungen der Mathematisch-Physischen Klasse der Königlich-Sächsischen Gesellschaft der Wissenschaften, vol. 32, no. 6, pp. 509-604, 1912.

[5] Z. Hashin and S. Shtrikman, "A variational approach to the theory of the effective magnetic permeability of multiphase materials," Journal of Applied Physics, vol. 33, no. 10, pp. 31253131, 1962.

[6] T. Mori and K. Tanaka, "Average stress in matrix and average elastic energy of materials with misfitting inclusions," Acta Metallurgica, vol. 21, no. 5, pp. 571-574, 1973.

[7] S. K. Kanaun and V. M. Levin, "Effective field method in mechanics of matrix composite materials," in Advances in Mathematical Modelling of Composite Materials, K. Z. Markov, Ed., pp. 1-58, World Scientific, 1994.

[8] K. Z. Markov, "Elementary micromechanics of heterogeneous media," in Heterogeneous Media: Micromechanics Modeling Methods and Simulations, K. Z. Markov and L. Preziozi, Eds., Modeling and Simulation in Science, Engineering and Technology, pp. 1-162, Birkhäuser, Boston, Mass, USA, 2000.

[9] R. L. Hamilton and O. K. Crosser, "Thermal conductivity of heterogeneous two-component systems," Industrial and Engineering Chemistry Fundamentals, vol. 1, no. 3, pp. 187-191, 1962.

[10] R. W. Zimmerman, "Effective conductivity of a twodimensional medium containing elliptical inhomogeneities," Proceedings of the Royal Society A: Mathematical, Physical and Engineering Sciences, vol. 452, no. 1950, pp. 1713-1727, 1996.

[11] P. L. Kapitza, “The study of heat transfer in helium II," Journal of Physics-USSR, vol. 4, pp. 177-181, 1941.

[12] S. K. Kanaun, "On singular models of a thin inclusion in a homogeneous elastic medium," Journal of Applied Mathematics and Mechanics, vol. 48, no. 1, pp. 50-58, 1984 (Russian).

[13] S. Torquato and M. D. Rintoul, "Effect of the interface on the properties of composite media," Physical Review Letters, vol. 75, no. 22, pp. 4067-4070, 1995.

[14] Y. Benveniste and T. Miloh, "The effective conductivity of composites with imperfect thermal contact at constituent interfaces," International Journal of Engineering Science, vol. 24, no. 9, pp. 1537-1552, 1986.

[15] Y. Benveniste, "Effective thermal conductivity of composites with a thermal contact resistance between the constituents: condilute case," Journal of Applied Physics, vol. 61, no. 8, pp. 2840-2843, 1987.

[16] Y. C. Chiew and E. D. Glandt, "Effective conductivity of dispersions: the effect of resistance at the particle surfaces," Chemical Engineering Science, vol. 42, no. 11, pp. 2677-2685, 1987.

[17] D. P. H. Hasselman and L. F. Johnson, "Effective thermal conductivity of composites with interfacial thermal barrier resistance," Journal of Composite Materials, vol. 21, no. 6, pp. 508-515, 1987.
[18] I. Sevostianov and M. Kachanov, "Effect of interphase layers on the overall elastic and conductive properties of matrix composites. Applications to nanosize inclusion," International Journal of Solids and Structures, vol. 44, no. 3-4, pp. 1304-1315, 2007.

[19] W. Jiajun and Y. Xiao-Su, "Effects of interfacial thermal barrier resistance and particle shape and size on the thermal conductivity of AlN/PI composites," Composites Science and Technology, vol. 64, no. 10-11, pp. 1623-1628, 2004.

[20] L. Shen and J. Li, "Homogenization of a fibre/sphere with an inhomogeneous interphase for the effective elastic moduli of composites," Proceedings of the Royal Society A: Mathematical, Physical and Engineering Sciences, vol. 461, no. 2057, pp. 14751504, 2005.

[21] T. Mütschele and R. Kirchheim, "Hydrogen as a probe for the average thickness of a grain boundary," Scripta Metallurgica, vol. 21, no. 8, pp. 1101-1104, 1987.

[22] V. M. Levin, "Determination of effective elastic-moduli of composite-materials," Soviet Physics-Doklady, vol. 220, pp. 1042-1045, 1975.

[23] S. K. Kanaun, "Effective field method in the linear problems of statics of composite media," Journal of Applied Mathematics and Mechanics, vol. 46, pp. 655-665, 1982.

[24] K. Hanada, K. Matsuzaki, and T. Sano, "Thermal properties of diamond particle-dispersed Cu composites," Journal of Materials Processing Technology, vol. 153-154, no. 1-3, pp. 514-518, 2004.

[25] S. Azeem and M. Zain-Ul-Abdein, "Investigation of thermal conductivity enhancement in bakelite-graphite particulate filled polymeric composite," International Journal of Engineering Science, vol. 52, pp. 30-40, 2012.

[26] Q. Kang, X. He, S. Ren et al., "Preparation of copper-diamond composites with chromium carbide coatings on diamond particles for heat sink applications," Applied Thermal Engineering, vol. 60, no. 1-2, pp. 423-429, 2013.

[27] W. Shen, W. Shao, Q. Wang, and M. Ma, “Thermal conductivity and thermal expansion coefficient of diamond $/ 5 \mathrm{wt} \% \mathrm{Si}-\mathrm{Cu}$ composite by vacuum hot pressing," Fusion Engineering and Design, vol. 85, no. 10-12, pp. 2237-2240, 2010.

[28] K. Raza and F. A. Khalid, "Optimization of sintering parameters for diamond-copper composites in conventional sintering and their thermal conductivity," Journal of Alloys and Compounds, vol. 615, pp. 111-118, 2014.

[29] Z. Ling, J. Chen, T. Xu, X. Fang, X. Gao, and Z. Zhang, “Thermal conductivity of an organic phase change material/expanded graphite composite across the phase change temperature range and a novel thermal conductivity model," Energy Conversion and Management, vol. 102, pp. 202-208, 2015. 

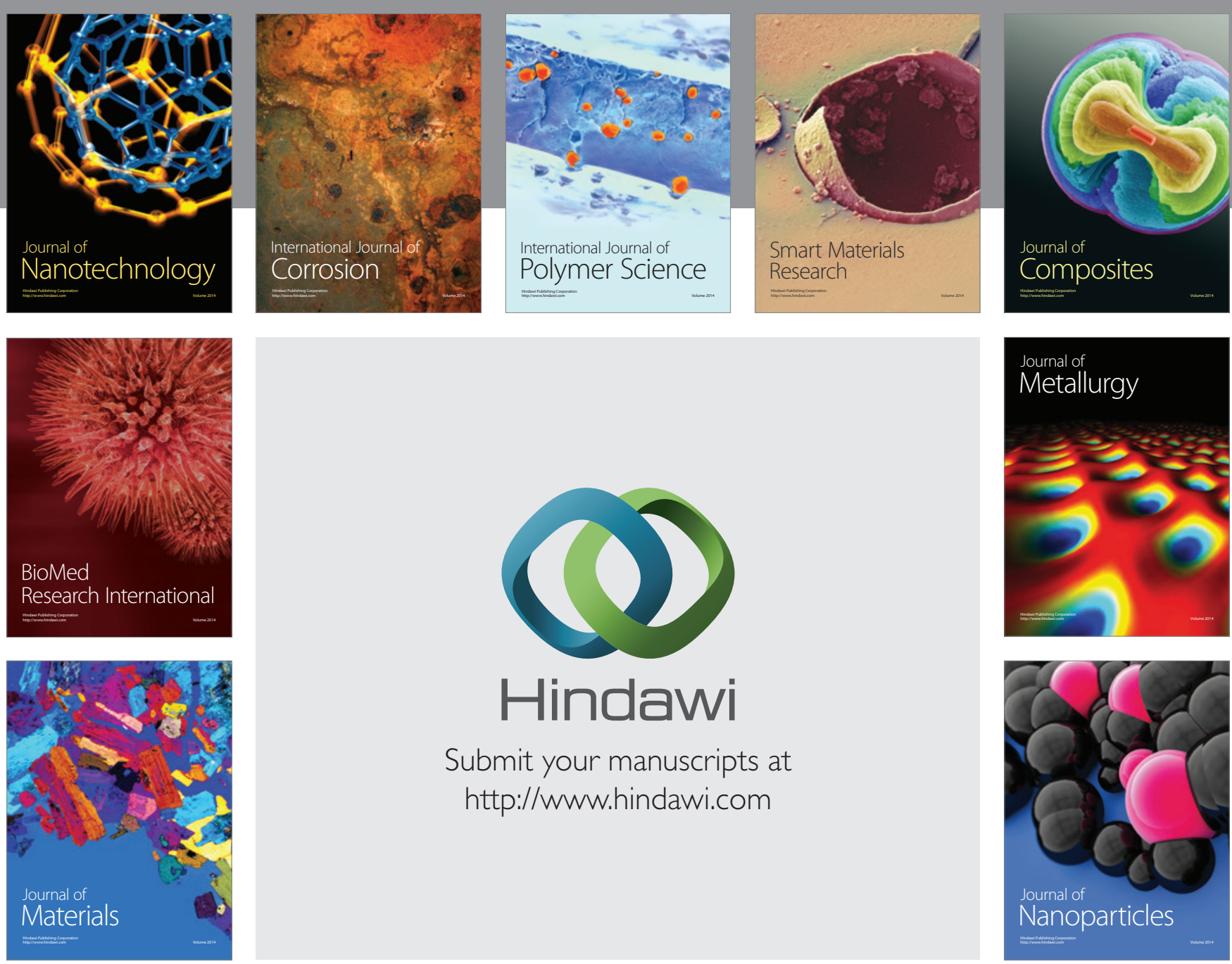

Submit your manuscripts at http://www.hindawi.com
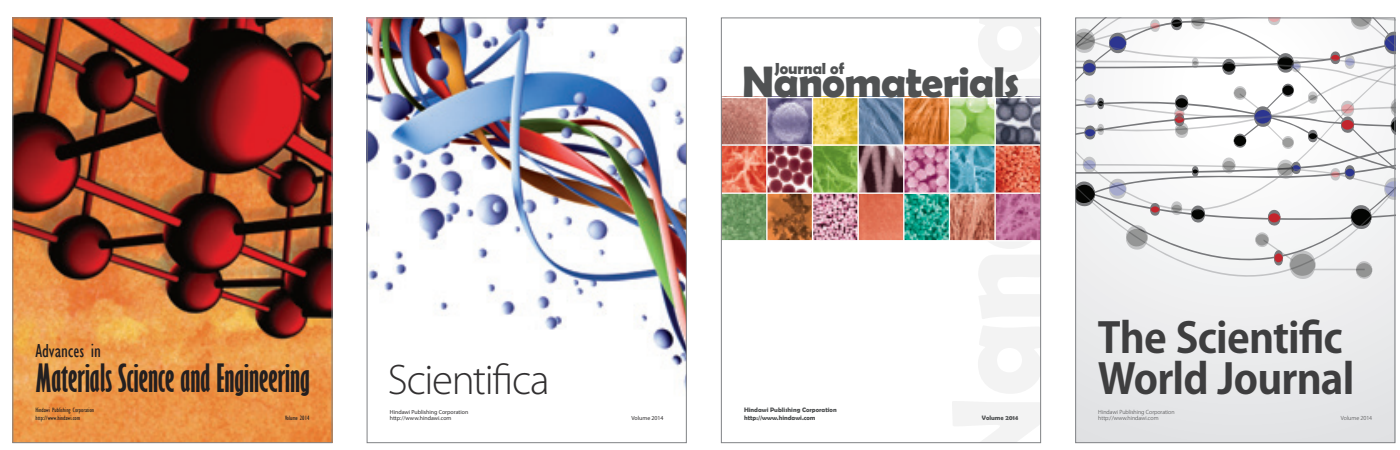

\section{The Scientific World Journal}
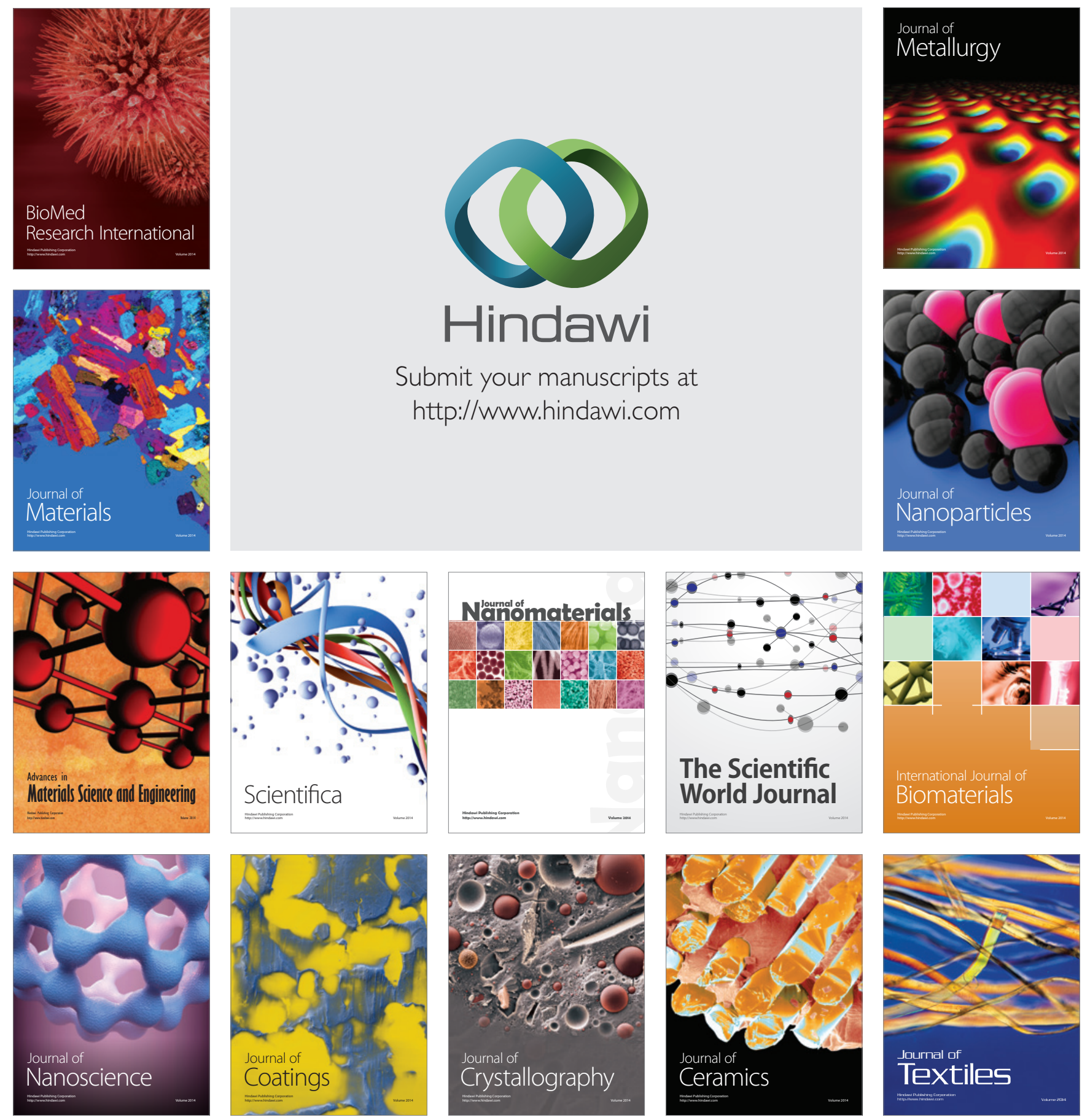